Military Technical College Kobry El-Kobbah, Cairo, Egypt

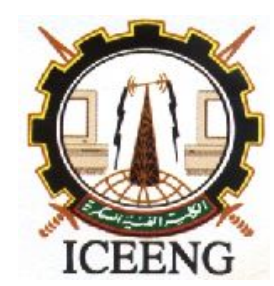

$9^{\text {th }}$ International Conference on Electrical Engineering ICEENG 2014

\title{
Performance Evaluation of Multimedia Applications QoS over Hybrid IPv4/MPLS Networks
}

\author{
By
}

\author{
Ahmed E. Fathy * \\ Hussien A. Elsaied **
}

\section{$\underline{\text { Abstract: }}$}

The demand for Multi-Protocol Label Switching (MPLS) is increasing day by day in most internet service provider networks. MPLS provides efficient forwarding, routing and switching of traffic flow through the network. MPLS technology has proven itself in providing the required Quality of service (QoS) needed by multimedia and real time application traffic, but it's almost impossible to convert the existing entire huge IPv4 networks to MPLS technology. This paper simulates hybrid IPv4/MPLS networks to overcome multimedia applications QoS problems by obtaining a performance close to pure MPLS network performance. Three different scenarios are investigated using OPNET simulator. Those scenarios are pure IP network, pure MPLS network, and hybrid IP/MPLS network. The simulation results point up the pros and cons of each scenario in terms of end-to-end delay, delay variation, packet loss ratio, packet delivery ratio, and voice MOS value. In short, pure MPLS network provides the best performance for multimedia traffic but close results can be achieved using hybrid IP/MPLS network.

\section{Keywords:}

MPLS, QoS, TE, MPLS TE, MPLS DiffServ TE

* Egyptian Armed Forces

** Ain Shams University 


\section{Introduction:}

Traditional IP routing protocols have several limitations like, but not limited to, scalability, traffic engineering support, and integration with Layer 2 backbones, which already exist in several service provider networks. With the rapid growth of the Internet and the establishment of IP as the Layer 3 protocol of choice in most environments, the drawbacks of traditional IP routing became more and more obvious.

MPLS is a modern technique for forwarding network data. In a MPLS network packets are assigned labels and the labels are used to make forwarding decisions without IP lookups at each node. It is called multi protocol because it supports any layer 3 network protocols. MPLS work between layer 2 and layer 3 which is called layer 2.5

technologies. MPLS provides the scalability for the Virtual private networks (VPNs) and support for end to end quality of service [1], [2].

MPLS technology came up to the picture to overcome the traditional IP routing and to make routing fast, manageable and able to carry heavy traffic, and accept new routing architectures. Several studies showed that MPLS has solved a lot of problems related to integration of layer 2 protocols [3], real time and multimedia application requirements [4], [5],[6] , fast rerouting and recovery [7], and traffic engineering [8],[9],[10]. In all of the previous studies, it's found that most of the simulated networks have only one type of traffic and for the networks that have more than one type of traffic, they have only one user for each traffic type. But there is a major problem to replace the entire huge number of existing IPv4 routers in the Internet to MPLS routers. This paper tries to provide a solution for this problem by integrating both of MPLS routers and IPv4 routers in the same network to gain the MPLS services with minimal change in the network. This paper simulates a large network with all types of traffic (voice - video data) with a large number of users. It contains eight subnets that include 300 VoIP users, 40 heavy load video users, and 80 data users. The rest of the paper is arranged as follows: Section 2 and 3 provide brief information about Quality of Service (QoS) and performance measures respectively. Simulation results are introduced in section 4 while section 5 concludes the paper.

\section{Quality of Service of MPLS}

Quality of Service is defined as the set of techniques to control bandwidth, delay, and jitter and packet loss in a network. QoS also provides techniques to supervise network traffic. It refers to a number of related features of telephony and computer networks that permits the transportation of traffic with the necessities. QoS manage when and how data is dropped when obstruction occurs through network administrators. At Label Edge 
Routers (LER) the Internet Protocol (IP) precedence is copied as Class of Service (CoS) and can be mapped to set the value of suitable MPLS CoS value in MPLS Label. Thus IP QoS is based on the IP precedence field in the IP header and MPLS QoS is based on the CoS bits in MPLS Label. Therefore MPLS CoS enables continuous IP QoS across the network [11], [12].

A queue scheduling allows constant output bandwidth by selecting the packet transmitted into the output queue. A queue scheduling is implemented on the output port of the routers. The packets are classified and queued for each output port of the routers. There are several scheduling methods are introduced by IETF but this paper focuses only on Weighted Fair Queuing (WFQ).The Weighted Fair Queuing is a flow based queuing algorithm designed to address limitations of the Fair Queuing (FQ) model. In this WFQ model, arriving packets are classified into flows and each flow is assigned to a First-In-First-Out (FIFO) queue. If all flows have the same priority/weight, WFQ effectively divides the interface bandwidth and distributed the bandwidth fairly among all the existing flows. For that reason, low-volume interactive flows are scheduled and not end up with packets waiting in their corresponding queues. High-volume interactive flows build up their corresponding queues and end up with packets waiting and delay more and possibility to drop packets. When the number of active flows exceeds the maximum number of dynamic queues, the new flows are assigned to the existing queues. As a result, multiple flows can end up sharing a queue [13].

\section{Performance Measures}

\section{A. Delay}

ITU-T Recommendation G.114 recommends the following one-way transmission time limits for connections with adequately controlled echo (complying with G.131) [14], [15]:

- 0 to $150 \mathrm{~ms}$ : acceptable for most user applications;

- 150 to 400 ms: acceptable for international connections;

- 400 ms: unacceptable for general network planning purposes.

\section{B. The E-Model}

The E-model defined in the ITU-T Rec G.107 [16] is an analytical model of voice quality used for network planning purposes. A basic result of the E-Model is the calculation of the R-factor which is a simple measure of voice quality ranging from a best case of 100 to a worst case of 50. The R-factor uniquely determines the Mean Opinion Score (MOS), which is the arithmetic average of opinion of voice quality as shown in table 1 [17]: 
Table (1): MOS Value

\begin{tabular}{|c|c|c|}
\hline R-Factor & Quality of Voice Rating & MOS \\
\hline $90<\mathrm{R}<100$ & Best & $4.34-4.5$ \\
\hline $80<\mathrm{R}<90$ & High & $4.03-4.34$ \\
\hline $70<\mathrm{R}<80$ & Medium & $3.6-4.03$ \\
\hline $60<\mathrm{R}<70$ & Low & $3.1-3.6$ \\
\hline $50<\mathrm{R}<60$ & Poor & $2.58-3.1$ \\
\hline
\end{tabular}

\section{Throughput Performance}

Throughput refers to the amount of data packets successfully received by the destination node. The throughput is usually measured in bits per second (bits/sec) and sometimes in data packets per second or data packets per time slot. A throughput with a higher value is more often an absolute choice in every network. The efficiency of a particular mechanism can be predicted by observing the overall throughput received by the network [13].

D. Packet Loss Ratio (PLR)

It is known that reliable traffic delivery is one of the main application requirements. The reliability index is PLR. The PLR index of any recipient can be defined as the ratio of the number of packets lost by some reason to the total number of packets transmitted.

\section{Simulations}

The aim of simulation is to study the performance of multimedia traffic in IPv4 network after converting some IPv4 routers into MPLS routers and compare the performance with both pure IPv4 network and pure MPLS network. And find out the effect of this replacement on the performance of the network.

Several network topologies have been simulated. Figure (1) shows a typical network topology which consists of core routers that provide connectivity to edge subnets, which are described later. The simulation is divided to two parts; and each part evaluates the network performance of three different scenarios of the network shown in figure (1). The network in the first scenario is pure IPv4 network, while the network in the second scenario is pure MPLS network. Both of IPv4 and MPLS are mixed in the third scenario. The difference between part 1 and part 2 is that the network load is increased, and more traffic engineering is applied.

This paper shows the enhancement of the multimedia performance over IPv4 network by converting some IPv4 routers from IP network to MPLS routers and using Label Switching Paths (LSPs) to carry voice and video traffic by comparing the results for the enhanced network with pure IPv4 network and pure MPLS network scenarios. 


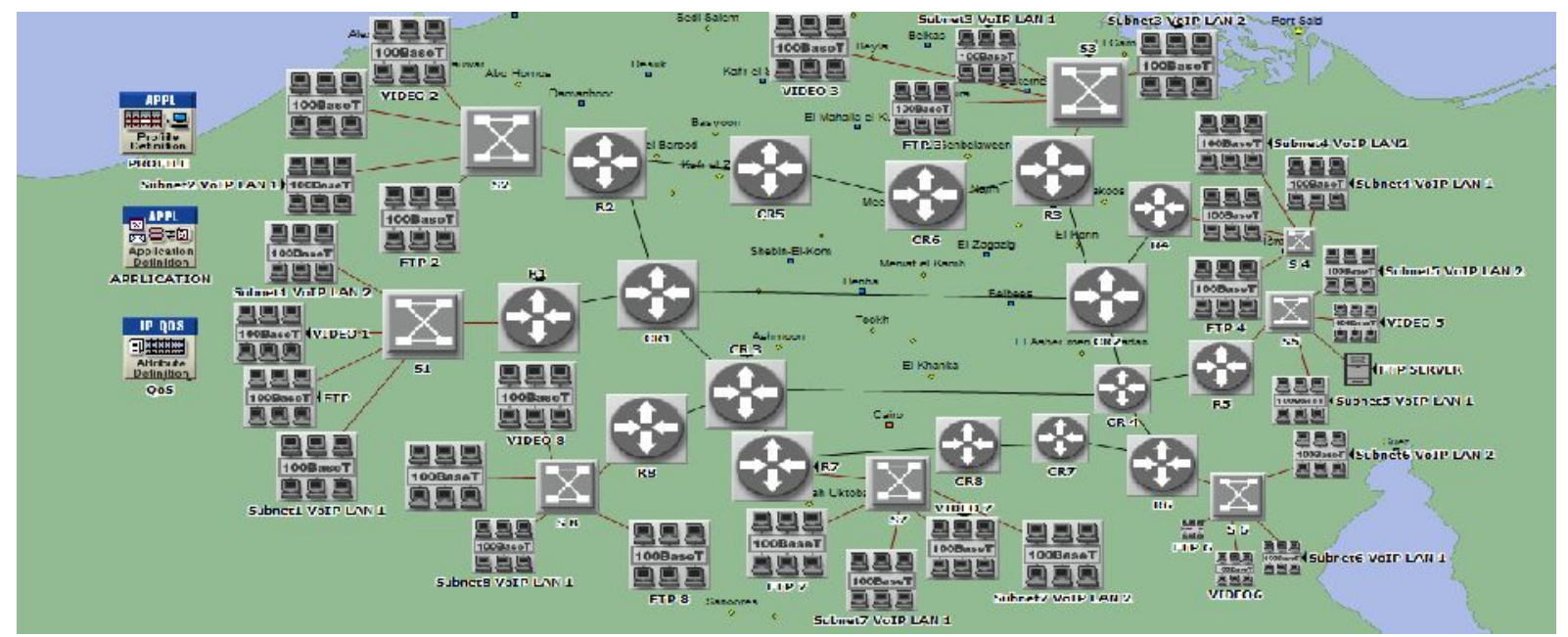

Figure (1): Simulation network

The network in figure (1) includes eight subnets; each subnet consists of two VoIP LANs, video LAN, and FTP LAN except subnet five which has FTP server instead of FTP LAN. Each subnet switch (S 1: S8) is a fast Ethernet switch. Full duplex fast Ethernet links are used to connect subnet LANs to switch; and OC3 links are used to connect routers to each others. Video and voice communications are between subnets (1) and (5), subnets (2) and (6), subnets (3) and (7), and subnets (4) and (8); while each FTP LAN communicates to FTP server in subnet (5). Table (2) shows all subnets LANs and the number of users per each LAN. The VoIP call is encoded using PCM codec with $64 \mathrm{~Kb} / \mathrm{s}$ (G.711), while FTP and video attributes are shown in table (3).

Table (2): Number of Users per LAN in Each Subnet

\begin{tabular}{|c|c||c|c|c|}
\hline \multirow{2}{*}{ Subnet } & \multirow{2}{*}{ LAN Name } & \multicolumn{3}{|c|}{ Scenario } \\
\cline { 3 - 5 } & IP Network & Mixed Network & $\begin{array}{c}\text { MPLS } \\
\text { Network }\end{array}$ \\
\hline \multirow{3}{*}{$\begin{array}{c}\text { Subnet } \\
\mathbf{1 , 2 , 3 , 4 , 6 , 7 , 8}\end{array}$} & VoIP LAN 1 & 10 & 10 & 10 \\
\cline { 2 - 5 } & VoIP LAN 2 & 15 & 15 & 15 \\
\cline { 2 - 5 } & VIDEO & 5 & 5 & 5 \\
\cline { 2 - 5 } Subnet 5 5 & VoIP LAN 1 & 10 & 10 & 10 \\
\cline { 2 - 5 } & VoIP LAN 2 & 15 & 10 & 15 \\
\cline { 2 - 5 } & VIDEO & 5 & 5 & 5 \\
\hline
\end{tabular}

Table (3): FTP and Video Attributes

\begin{tabular}{|c|c||c|c|}
\hline \multicolumn{2}{|c||}{ FTP Attributes } & \multicolumn{2}{c|}{ VIDEO Conferencing Attributes } \\
\hline Attribute & Value & Attribute & value \\
\hline $\begin{array}{c}\text { Command Mix } \\
\text { (Get/Total) }\end{array}$ & $50 \%$ & $\begin{array}{c}\text { Frame Inter-arrival } \\
\text { Time }\end{array}$ & 15 frames / sec \\
\hline
\end{tabular}




\begin{tabular}{|c|c||c|c|}
\hline $\begin{array}{c}\text { Inter-Request Time } \\
\text { (seconds) }\end{array}$ & Constant (30) & Frame size & $128 \times 240$ pixels \\
\hline File size (bytes) & $\begin{array}{c}\text { Constant } \\
(5,000,000)\end{array}$ & Type of Service & $\begin{array}{c}\text { Interactive } \\
\text { Multimedia (5) }\end{array}$ \\
\hline Type of Service & Best Effort (0) & & \\
\hline
\end{tabular}

\section{A. Part One}

The first scenario is pure IPv4 network. Routers R1 to R8, Provider Edge, are the interface between the eight subnets and the core routers, while routers CR1 to CR8 are the core routers.

The second scenario is pure MPLS network. Routers R1 to R8 are LERs where subnets voice and video traffic are mapped into LSPs, while CR1 to CR8 are Label Switching Routers (LSRs). In this scenario eight LSPs are used (R1-R5, R5-R1, R2-R6, R6-R2, R3-R7, R7-R3, R4-R8 and R8-R4).

The third one is hybrid IPv4/MPLS network in which CR1, CR2, CR3 and CR4 routers are converted to MPLS routers. CR1 router is the LER of subnets 1 and 2, CR2 router is the LER of subnets 3 and 4, CR3 router is the LER of subnets 7 and 8, and CR4 router is the LER of subnets 5 and 6.

In all of the three scenarios, DiffServ and WFQ are applied. The simulation time is selected to be big enough to reach steady state results, which is 300 seconds.

\section{1)Video results}

Figures (2) and (3) show the packet delay variation, end to end delay (in seconds) and the received traffic in bytes for video traffic, while table (4) shows the average packet delay variation and end to end delay for all networks. Table (5) shows Total video packet sent, total packet received and packet loss ratio.

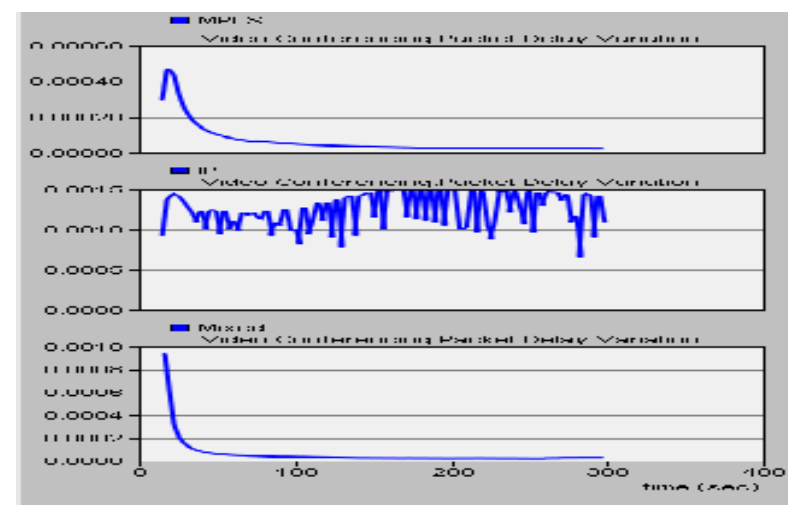

(a)

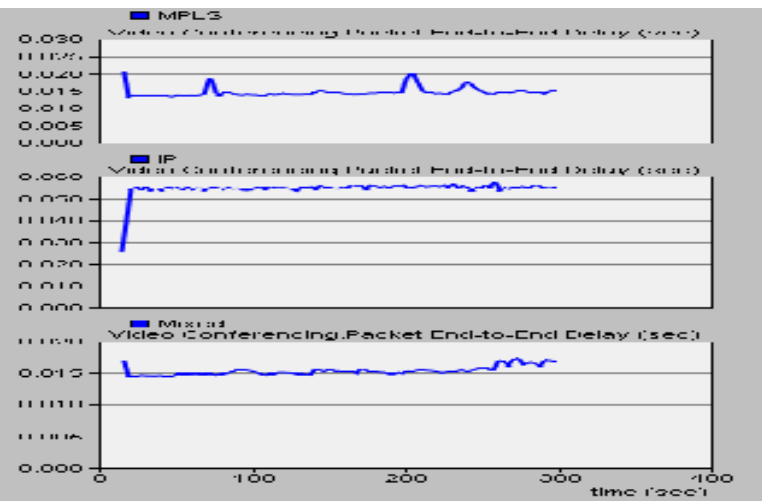

(b)

Figure (2): MPLS, IP, and mixed network video results. (a) Packet Delay Variation, (b) Packet End-to-End Delay. 
Table (4): Average Packet Delay Variation and Average End-to-End Delay

\begin{tabular}{|c|c|c|}
\hline Scenario & $\begin{array}{c}\text { Average Packet Delay } \\
\text { Variation }\end{array}$ & $\begin{array}{c}\text { Average End-to-End } \\
\text { Delay }\end{array}$ \\
\hline IP & $1.2 \mathrm{~ms}$ & $55 \mathrm{~ms}$ \\
\hline MPLS & $30 \mu \mathrm{s}$ & $14 \mathrm{~ms}$ \\
\hline Mixed & $30 \mu \mathrm{s}$ & $15 \mathrm{~ms}$ \\
\hline
\end{tabular}

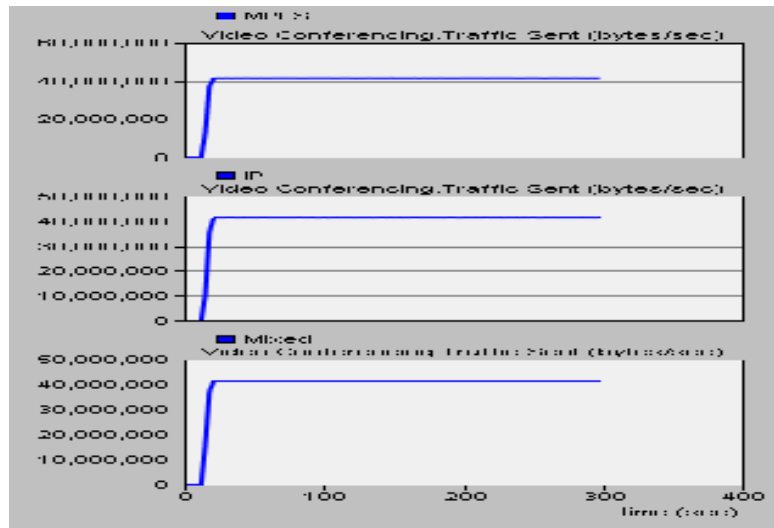

(a)

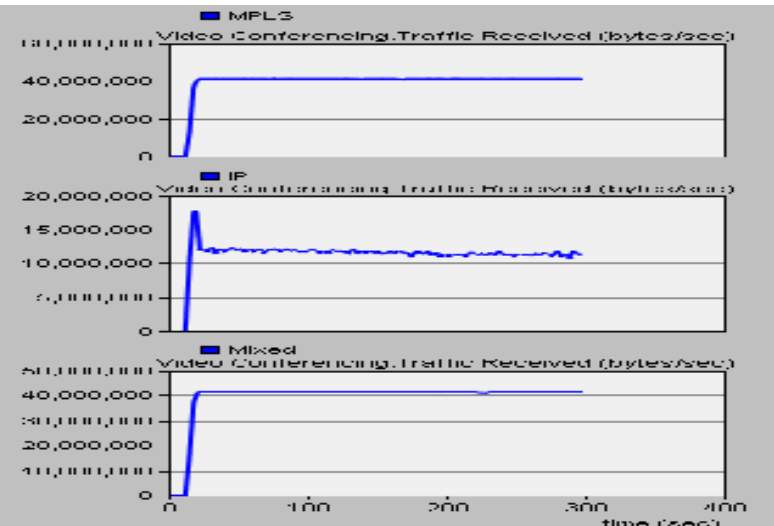

(b)

Figure (3): Video traffic in bytes. (a) Traffic Sent, (b) Traffic Received.

Table (5): Video Packet Loss Ratio

\begin{tabular}{|c|c|c|c|}
\hline & MPLS & IP & Mixed \\
\hline Total Packet Sent & 339084 & 338697 & 339335 \\
\hline $\begin{array}{c}\text { Total Packet } \\
\text { Received }\end{array}$ & 338973 & 96643 & 339126 \\
\hline Packet Lost & 111 & 242054 & 209 \\
\hline $\begin{array}{c}\text { Packet Loss Ratio } \\
\%\end{array}$ & 0.0327 & 71.466 & 0.0616 \\
\hline $\begin{array}{c}\text { Packet Delivery } \\
\text { Ratio \% }\end{array}$ & 99.967 & 28.534 & 99.938 \\
\hline
\end{tabular}

Figures (2), and (3) and Tables (4), and (5) Show that MPLS network has the best video performance. Also mixed network video results are better than IP network and gives results close to MPLS network results.

2) Voice Results

Figure (4) show voice packet delay variation and end to end delay (in seconds). Table (6) shows MOS value, average packet delay variation and average end to 
end delay, while table (7) shows total packet sent, total packet received and packet loss ratio.

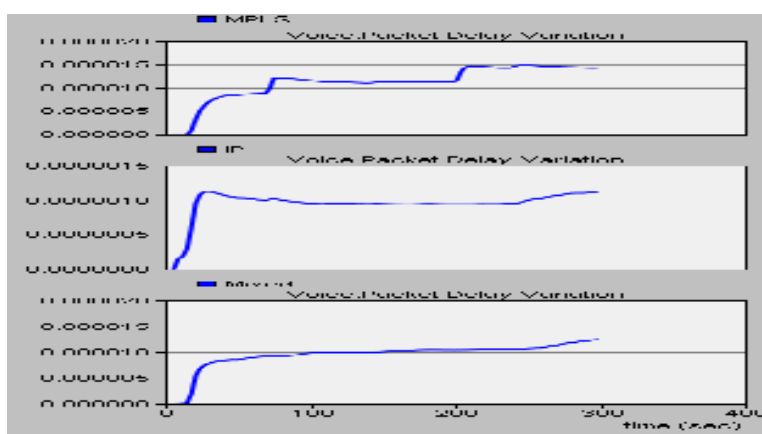

(a)

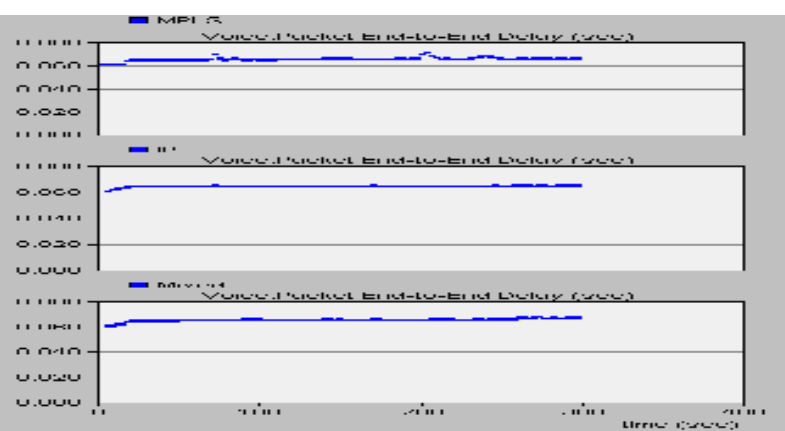

(b)

Figure (4): MPLS, IP, and mixed network voice results. (a) Packet Delay Variation, (b) Packet End-to-End Delay.

Table (6): MOS Value, Average Packet Delay Variation and Average End-to-End Delay

\begin{tabular}{|c|c|c|c|}
\hline Scenario & MOS Value & $\begin{array}{c}\text { Average Packet Delay } \\
\text { Variation }\end{array}$ & $\begin{array}{c}\text { Average End-to-End } \\
\text { Delay }\end{array}$ \\
\hline IP & 3.680 & $1 \mu \mathrm{s}$ & $65 \mathrm{~ms}$ \\
\hline MPLS & 3.687 & $11.5 \mu \mathrm{s}$ & $66 \mathrm{~ms}$ \\
\hline Mixed & 3.6865 & $10.5 \mu \mathrm{s}$ & $66 \mathrm{~ms}$ \\
\hline
\end{tabular}

Table (7): Video Packet Loss Ratio

\begin{tabular}{|c|c|c|c|}
\hline & MPLS & IP & Mixed \\
\hline Total Packet Sent & 6393336 & 6381650 & 6380264 \\
\hline $\begin{array}{c}\text { Total Packet } \\
\text { Received }\end{array}$ & 6393109 & 6205504 & 6214444 \\
\hline Packet Lost & 227 & 176146 & 165820 \\
\hline $\begin{array}{c}\text { Packet Loss Ratio } \\
\%\end{array}$ & 0.0035 & 2.76 & 2.5 \\
\hline $\begin{array}{c}\text { Packet Delivery } \\
\text { Ratio \% }\end{array}$ & 99.996 & 97.24 & 97.5 \\
\hline
\end{tabular}

Figure (4) and table (7) Show that MPLS network gives the best performance for voice in throughput and PLR. Also mixed network is better than IP network, but table (6) shows that IP network has the lowest average packet delay variation. However, MPLS and mixed networks end-to-end delay results are accepted and give the required QoS. This part of the paper shows that enhancing the IPv4 network by converting some IP routers to MPLS routers enhances multimedia traffic performance to a degree near to 
that is given by MPLS network. From table (4) and (5) it is found that in the pure IPv4 network the video traffic average packet delay variation is $1.2 \mathrm{~ms}$, the average end-toend delay is $55 \mathrm{~ms}$ and PLR ratio is $71.466 \%$, while in MPLS network the average packet delay variation is $30 \mu \mathrm{s}$, the average end-to-end delay is $14 \mathrm{~ms}$ and PLR ratio is $0.0327 \%$,. In the mixed network the average packet delay variation is $30 \mu \mathrm{s}$, the average end-to-end delay is $15 \mathrm{~ms}$ and PLR ratio is $0.0616 \%$ which is close to the corresponding values in the case of pure MPLS network. Also, the voice PLR ratio is $2.76 \%, 0.0035 \%$, and $2.5 \%$ in IPv4, MPLS, and mixed networks respectively.

\section{B. Part 2}

In this part all network traffic load is increased by adding additional voice LANs to subnets 2, 3, 6 and 8. Each additional LAN has 25 VoIP users. Also simulation time is 300 seconds.

\section{1)Video results}

Figures (5) and (6) show the packet delay variation, end to end delay (in seconds) and the received traffic in packets per second for video traffic, while table 8 shows the average packet delay variation and end to end delay for all networks. Table 9 shows Total video packet sent, total packet received and packet loss ratio.

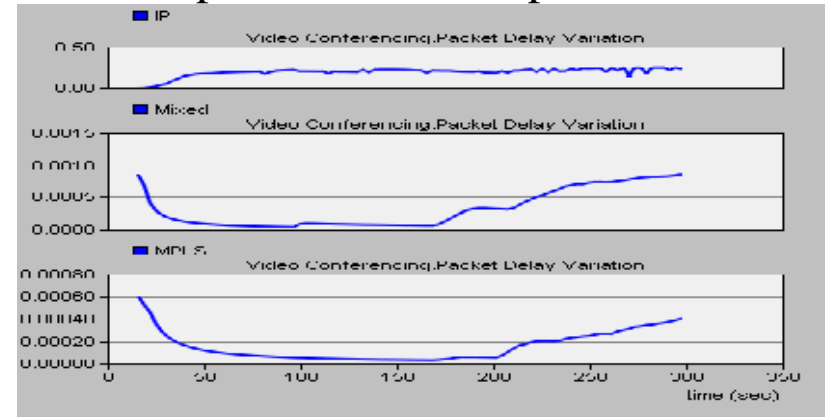

(a)

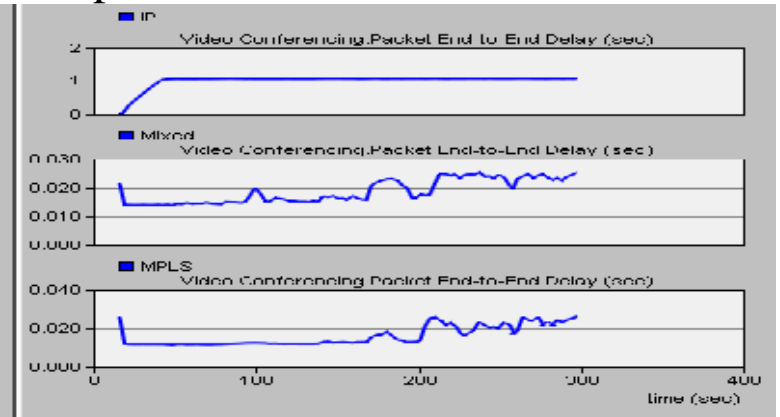

(b)

Figure (5): MPLS, IP, and mixed network video results. (a) Packet Delay Variation, (b) End-to-End Delay

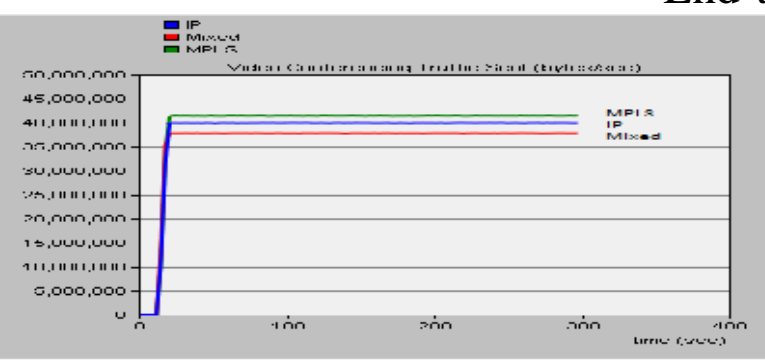

(a)

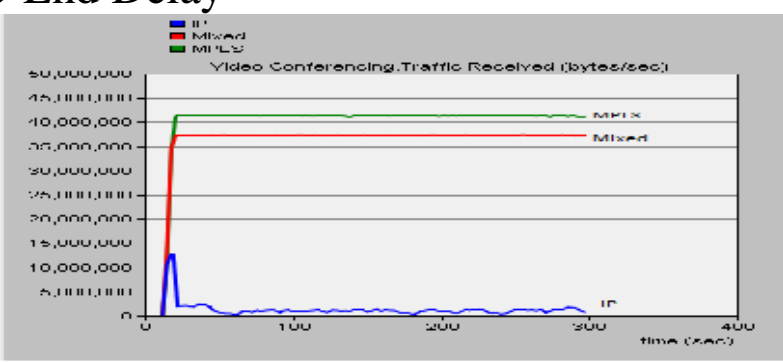

(b)

Figure (6): Video conference traffic. (a) Video Packet Sent, (b) Video Packet Received 
Table (8): Average Packet Delay Variation and Average End-to-End Delay

\begin{tabular}{|c|c|c|}
\hline Scenario & $\begin{array}{c}\text { Average Packet Delay } \\
\text { Variation }\end{array}$ & $\begin{array}{c}\text { Average End-to-End } \\
\text { Delay }\end{array}$ \\
\hline IP & $194.4 \mathrm{~ms}$ & $1 \mathrm{~s}$ \\
\hline MPLS & $153 \mu \mathrm{s}$ & $16.1 \mathrm{~ms}$ \\
\hline Mixed & $316 \mu \mathrm{s}$ & $18.5 \mathrm{~ms}$ \\
\hline
\end{tabular}

Table (9): Video Packet Loss Ratio

\begin{tabular}{|c|c|c|c|}
\hline & MPLS & IP & Mixed \\
\hline Total Packet Sent & 338586 & 325840 & 309674 \\
\hline $\begin{array}{c}\text { Total Packet } \\
\text { Received }\end{array}$ & 338186 & 11275 & 305209 \\
\hline Packet Lost & 400 & 314565 & 4456 \\
\hline $\begin{array}{c}\text { Packet Loss Ratio } \\
\%\end{array}$ & 0.118 & 96.539 & 1.442 \\
\hline $\begin{array}{c}\text { Packet Delivery } \\
\text { Ratio }\end{array}$ & 99.882 & 3.46 & 98.558 \\
\hline
\end{tabular}

2) Voice Results

Figure (7) shows the packet delay variation and end to end delay (in seconds). Table 10 shows MOS value, average packet delay variation and average end to end delay, while table 11 shows total packet sent, total packet received and packet loss ratio.

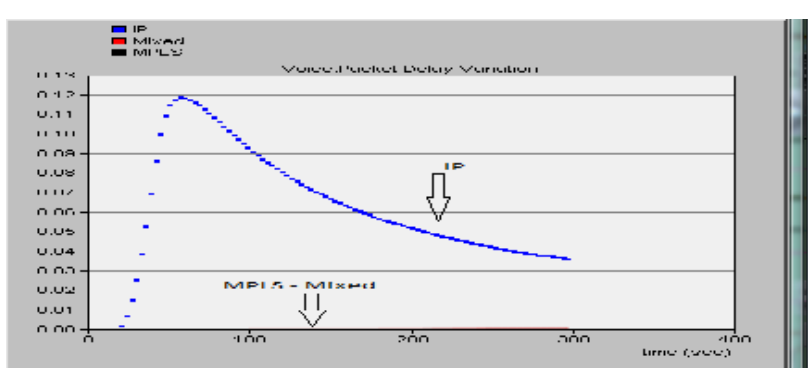

(a)

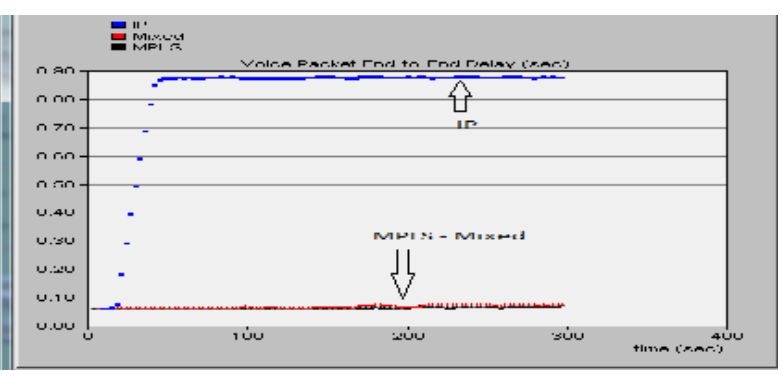

(b)

Figure (7): MPLS, IP, and mixed network voice results. (a) Packet Delay Variation, (b) Packet End-to-End Delay. 
Table (10): MOS Value, Average Packet Delay Variation and Average End-to-End Delay

\begin{tabular}{|c|c|c|c|}
\hline Scenario & MOS Value & $\begin{array}{c}\text { Average Packet Delay } \\
\text { Variation }\end{array}$ & $\begin{array}{c}\text { Average End-to-End } \\
\text { Delay }\end{array}$ \\
\hline IP & 3.688 & $64 \mathrm{~ms}$ & $0.84 \mathrm{~s}$ \\
\hline MPLS & 3.688 & $15.5 \mu \mathrm{s}$ & $66 \mathrm{~ms}$ \\
\hline Mixed & 3.688 & $50 \mu \mathrm{s}$ & $70.7 \mathrm{~ms}$ \\
\hline
\end{tabular}

Table (11): Voice Packet Loss Ratio

\begin{tabular}{|c|c|c|c|}
\hline & MPLS & IP & Mixed \\
\hline Total Packet Sent & 9471620 & 9454204 & 9455500 \\
\hline Total Packet Received & 9470221 & 9051749 & 9216786 \\
\hline Packet Lost & 1399 & 402455 & 328714 \\
\hline Packet Loss Ratio \% & 0.01477 & 4.257 & 2.5 \\
\hline Packet Delivery Ratio \% & 99.98523 & 95.743 & 97.5 \\
\hline
\end{tabular}

This part of the paper shows that enhancing the IPv4 network by converting some IP routers to MPLS routers enhances multimedia traffic performance to a degree near to that is given by MPLS network. From table (8) and (9) it is found that in the pure IP4 network the video traffic average packet delay variation is $194.4 \mathrm{~ms}$, the average end-toend delay is 1s and PLR ratio is $96.539 \%$, while in MPLS network the average packet delay variation is $153 \mu \mathrm{s}$, the average end-to-end delay is $16.1 \mathrm{~ms}$ and PLR ratio is $0.118 \%$. In the mixed network the average packet delay variation is $316 \mu \mathrm{s}$, the average end-to-end delay is $18.5 \mathrm{~ms}$ and PLR ratio is $1.442 \%$ which is close to the corresponding values in the case of pure MPLS network. From tables (10) and (11) it is found that in the full IP4 network the voice traffic average packet delay variation is 64 $\mathrm{ms}$, the average end-to-end delay is $0.84 \mathrm{~s}$ and PLR ratio is $4.257 \%$, while in MPLS network the average packet delay variation is $15.5 \mu \mathrm{s}$, the average end-to-end delay is $66 \mathrm{~ms}$ and PLR ratio is $0.01477 \%$, In the mixed network the average packet delay variation is $50 \mu \mathrm{s}$, the average end-to-end delay is $70.7 \mathrm{~ms}$ and PLR ratio is $2.5 \%$ which is close to the corresponding values in the case of full MPLS network.

\section{Conclusion}

This paper addressed the QoS of multimedia traffic by simulating three different scenarios, which are pure IP network, pure MPLS network, and IP/MPLS hybrid network. MPLS network gives better performance for multimedia traffic than IPv4 network, which is expected. But it's hard to replace the existing IPv4 routers in the Internet with MPLS routers. This paper discussed the network performance details to solve this problem and showed that IPv4 network performance can be enhanced by 
converting selected IP routers to MPLS routers instead of using completely new MPLS network. By using LSPs through the converted MPLS routers in IPv4 network to carry multimedia traffic, the performance is enhanced to a degree near to that of MPLS network. From part one simulation; the mixed network has better results for video and voice communication than pure IPv4 network and near to that given in pure MPLS network. In part two with the extra load, MPLS network and mixed network can provide the requirements for multimedia traffic because of their scalability and the efficient use of traffic engineering.

\section{REFERENCES}

[1] Narinder Kaur, Raj Kumar, and Bharti Gupta, "Interpretation of MPLS enabled network with QoS model," International Journal of Innovations in Engineering and Technology (IJIET), Vol. 2, Issue1, pp. 130-136, Feb. 2013. [2] Rashed Q. Shawl, Rukhsana Thaker, and Er. Jasvinder Singh, "A Review: Multi Protocol Label Switching (Mpls)", Int. Journal of Engineering Research and Applications, Vol. 4, Issue 1(Version 2), pp.66-70, Jan. 2014.

[3] Cemal Kocak, Ismail Erturk, and HuseyinEkiz. "MPLS over ATM and IP over ATM methods for multimedia applications," Journal Computer Standards \& Interfaces, Vol. 31, Issue 1, pp. 153-160, January 2009.

[4] Md. Tariq Aziz, Mohammad Saiful Islam, Md. Nazmul Islam khan, and Prof. Adrian Popescu, "Effect of packet delay variation on video/voice over DiffServ-MPLS in IP/IPv6 networks," International Journal of Distributed and Parallel Systems (IJDPS) Vol.3, No.1, pp. 27-47, January 2012.

[5] Masoomeh Torabzadeh, and WessamAjib. "A short-time burst degradation classifier for real-time traffic with application in MPLS ingress nodes," Journal of Communications, vol. 6, No.3, pp. 215-224, April 2011.

[6] Ruey-Shun Chen, Y. Tsai, K.C. Yeh, and H.Y. Chen, "Using policy-based MPLS management architecture to improve QoS on IP network," WSEAS Transactions on Computers, Vol. 7, Issue 5, pp. 341-350, May 2008.

[7] Sabri M. Hanshi, and Wajdi Al- Khateeb, "Enhancing QoS protection in MPLS networks," Second International Conference on Network Applications (NETAPPS), Protocols and Services IEEE Computer Society, Kedah, pp. 95100, September 2010.

[8] Prabhjot Kaur, and Satish Devane, "Load balancing in MPLS," International Journal of Computer Engineering \& Science (IJCES), pp. 107-117, Nov. 2013

[9] Mohammad M. Shahsavar, and Adnan A. Al-Tunsi. "MPLS performance modeling using traffic engineering to improve QoS routing on IP networks," SoutheastCon, Columbia, SC, Proceedings IEEE, pp. 152-157, Apr. 2002. [10] Mahesh Kr. Porwal, Anjulata Yadav, and S. V. Charhate, "Traffic analysis of MPLS and non MPLS network including MPLS signaling protocols and traffic distribution in OSPF and MPLS," Emerging Trends in Engineering and Technology, ICETET'08.First International Conference, Nagpur, Maharashtra, pp.187-192, July 2008.

[11]Jitendra Joshi, Sonali Gupta, Priti Gupta, Nisha Singh, and ManjariKumari, "Multi-protocol label switching with quality of service in high speed computer network," International Journal of Engineering Science and Innovative Technology (IJESIT), Volume 2, Issue 2, pp. 83-87, March 2013.

[12] K. Dhuri, and Alam Shaikh, "Review on QoS Improvement with MPLS Mechanism in NGN," International Journal of Innovative Research in Science, Engineering and Technology, Vol. 3, Issue 2, pp. 9431-9438, Feb 2014. [13] Md. T. Aziz, "Performance evaluation of real-time applications over DiffServ/MPLS in IPv4/IPv6 networks," Master Thesis, School of Computing Blekinge, Institute of Technology 37179 Karlskrona Sweden, May 2011.

[14] Bur Goode, "Voice over internet protocol (VoIP)," Proceedings of the IEEE vol. 90, Issue 9, pp.1495-1517, September 2002.

[15] ITU-T Recommendation G.114, “One-way transmission time,” Sep. 2003.

[16] ITU-T Rec. G. 107, "The E-Model, a computational model for use in transmission planning," 2003.

[17] Robert G. Cole, and Joshua H. Rosenbluth, "Voice over IP performance monitoring," Journal Computer Communication Review (CCR), vol. 31,no. 2, pp. 9-24, 2001. 